\title{
Rechts- und Staatsphilosophie bei G.W. Leibniz
}

\author{
Herausgegeben von Tilmann Altwicker, Francis Cheneval und Matthias Mahlmann
}

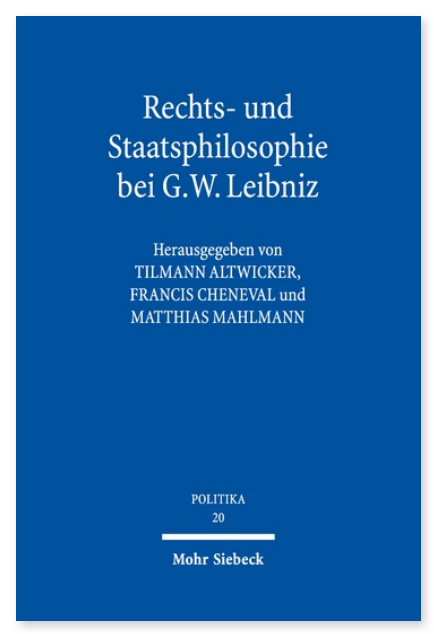

2020. XI, 350 Seiten. POLITIKA 20

ISBN 978-3-16-156968-5 fadengeheftete Broschur 49,00€ ISBN 978-3-16-156969-2 eBook PDF 49,00€
Der Band beleuchtet Leibniz' Rechts- und Staatsphilosophie im Kontext seiner Metaphysik, Logik, Erkenntnistheorie und Moralphilosophie. Auch die Rezeption seiner Rechts- und Staatsphilosophie wird in den Beiträgen reflektiert. Gerade im Hinblick auf die aktuelle Diskussion um die politische Gestaltung Europas und die kosmopolitische Gestaltung der Globalisierung verdient seine Philosophie Aufmerksamkeit - nicht zuletzt auch auf Grund interner Spannungen, die das politische Selbstverständnis Europas bis heute kennzeichnen.

Inhaltsübersicht

\section{Teil 1: Recht und Erkenntnistheorie}

Ursula Goldenbaum: Grounding Jurisprudence in Theology - Leibniz's Rebuff of Protestant Voluntarism - Andreas Blank: Presumptions and Cognitive Simplicity in Leibniz and Early Modern Legal Theory - Christian Barth: Interrelations between Leibniz's Theoretical Philosophy and his Philosophy of Law: Leibniz on Human and Divine Cognition - Stephan Schmid: Leibniz' metaphysische Begründung des Rechts. Kommentare zu den Beiträgen von Goldenbaum, Blank und Barth

\section{Teil 2: Moralphilosophie}

Hubertus Busche: Zur Leistungsfähigkeit des Leibnizschen Naturrechts - Matthias Mahlmann: Die geistige Wurzel der Gerechtigkeit - Rationalismus und Epistemologie in Leibniz' praktischer Philosophie - Alexander Aichele: Ponderierte Indifferenz? Der Indeterminismus in Leibniz' Labyrinth der Willensfreiheit - Katja Stoppenbrink: Auf dem Weg zur Autonomie: Leibniz und Pufendorf - zu den Gründen moralischer Verpflichtung - Tobias Herbst: Leibniz' Moralphilosophie. Kommentare zu den Beiträgen von Busche, Mahlmann, Aichele und Stoppenbrink

\section{Teil 3: Naturrecht}

Matthias Armgardt: Inhalte des Naturrechts bei Leibniz - Lorenz Kähler: Billigkeit versus Recht bei Leibniz - Rainer Keil: Recht und Billigkeit; Eigentum, insbesondere an Menschen, Widerstandsrecht, Innoxia Utilitas: Kommentierung zweier Beiträge zum Naturrecht bei Leibniz

Teil 4: Staat und Internationale Beziehungen

Francis Cheneval: Leibniz' Staats- und Europakonzept - Detlef von Daniels: Vom Kosmopolitismus zur Leibniz'schen Metaphysik und wieder zurück - Tilmann Altwicker: Völkerrechtsmetaphysik bei G. W. Leibniz. Versuch einer Überwindung der Universalismus-/Partikularismus-Dichotomie - Thomas Kleinlein: G. W. Leibniz als Völkerrechtsdenker: Kommentar

\section{Teil 5: Rezeption}

Stephan Meder: Leibniz' Rezeption durch Friedrich Carl von Savigny und Otto von Gierke - Christoph-Eric Mecke: Die Rezeption des Rechtsdenkens von Gottfried Wilhelm Leibniz bei Gustav Hugo (1764-1844) und Rudolf von Jhering (1818-1892) - Elisabetta Fiocchi Malaspina: Reading Leibniz through Emer de Vattel: Défense du système leibnitien (1741) - Stephan Waldhoff: »lch habe von meiner ersten jugend an ... mein gemüth auff ...gemeines beste gerichtet«. Gottfried Wilhelm Leibniz - eine politisch-biographische Skizze

Tilmann Altwicker ist Inhaber einer Förderungsprofessur des Schweizerischen Nationalfonds (SNF) für Öffentliches Recht, Völkerrecht, Rechtsphilosophie und Empirische Rechtsforschung an der Universität Zürich.

Francis Cheneval ist Inhaber des Lehrstuhls für Politische Philosophie an der Universität Zürich.

Matthias Mahlmann ist Professor für Philosophie und Theorie des Rechts, Rechtssoziologie und Internationales Öffentliches Recht an der Universität Zürich.

Jetzt bestellen:

https://www.mohrsiebeck.com/buch/rechts-und-staatsphilosophie-bei-gw-leibniz-9783161569685?no_cache=1 order@mohrsiebeck.com

Telefon: +49 (0)7071-923-17

Telefax: $+49(0) 7071-51104$ 